\title{
Aluminum modulation of proteolytic activities
}

\author{
Giulio Lupidi ${ }^{a}$, Mauro Angeletti ${ }^{a}$, Anna Maria Eleuteri ${ }^{a}$, Evandro Fioretti ${ }^{\mathrm{a}, *}$, \\ Stefano Marini ${ }^{b}$, Magda Gioia ${ }^{b}$, Massimo Coletta ${ }^{b}$ \\ a Dipartimento di Biologia Molecolare, Scuola di Specializzazione in Biochimica Clinica, Cellulare ed Animale, Università di Camerino, 62032 Camerino \\ (MC), Italy \\ ${ }^{\mathrm{b}}$ Dipartimento di Medicina Sperimentale e Scienze Biomediche, Università di Roma Tor Vergata, Via di Tor Vergata 135, I-00133 Rome, Italy
}

Received 14 August 2001; accepted 4 March 2002

\section{Contents}

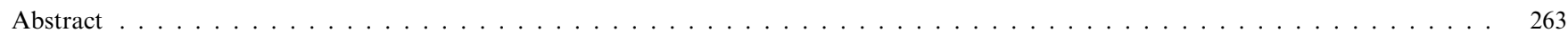



2. Effects of aluminum on the biological activity of serine proteinases $\ldots \ldots \ldots \ldots$

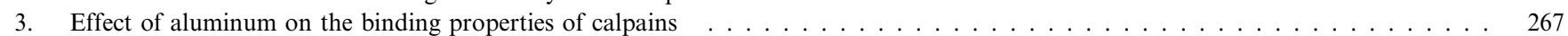

4. Effect of aluminum on the proteolytic enzymes of cerebral cortex $\ldots \ldots \ldots \ldots \ldots$

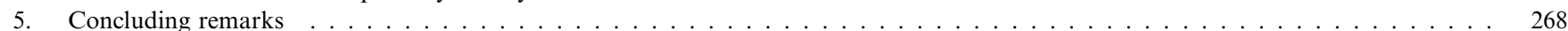

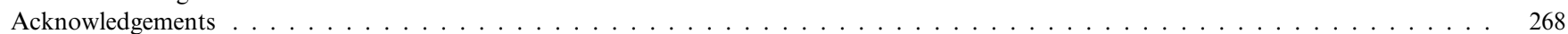

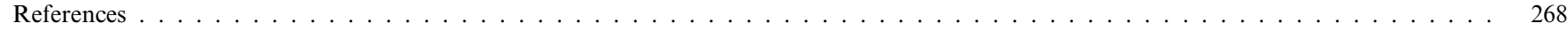

\begin{abstract}
The effect of aluminum ions on the activity of proteolyic activities, mainly serine proteases and calpains, has been examined. Aluminum affects the biological activity of proteolytic activities either through a direct effect on the enzyme molecule or through a deregulation of the control mechanisms acting on them. Binding of the ion, most likely results in molecular rearrangements affecting both the substrates binding site and the site involved in the recognition of macromolecular inhibitors. As whole, the data reported clearly indicate that aluminum significatively affects the intracellular protein turnover, most likely triggering catastrophic events for the cellular life. The physiopathological significance of these effects has been discussed, in particular for neurological disorders (the Alzheimer's disease included) where an imbalance of proteolytic as well as antiproteolytic systems appears a crucial event both for the formation of neuritic plaques and neurofibrillary tangles which are the major hallmarks of the disease. (C) 2002 Elsevier Science B.V. All rights reserved.
\end{abstract}

Keywords: Aluminum modulation; Proteolytic activities; Aluminum ions

\section{Introduction}

Aluminum is a tin-white, malleable, ductile metal, odourless element, with a melting point of $660{ }^{\circ} \mathrm{C}$, soluble in alkalis, hydrochloric and sulphuric acid. It is the most abundant metal of the earth crust and the third most abundant element after oxygen and silicon, occurs only in combined form and it is able to reduce the

\footnotetext{
* Corresponding author. Tel.: +39-737-403210; fax: +39-737403350.

E-mail address: evandro.fioretti@unicam.it (E. Fioretti).
}

cations of many heavy metals to the metallic state. Aluminum can be found in living cells or biological fluids $[1,2]$, both in free (mononuclear ions) or complexed form (low- or high-molecular weight complexes). In aqueous media, in fact, aluminum can be distributed in several soluble species, depending on the $\mathrm{pH}$ value, and it shows a preferential interaction with oxygen donor ligands (i.e. carboxylate and phosphate groups which in biological systems are responsible for the formation of complexes) [3]. The ionic radius of $\mathrm{Al}^{3+}$ is very similar to that of $\mathrm{Mg}^{2+}$ and $\mathrm{Fe}^{3+}$ and the substitution by $\mathrm{Al}^{3+}$ in $\mathrm{Mg}^{2+}$-depending enzymes causes their inhibition [1,2]. The aluminum transport 
occurs through the plasma, where the ion can bind citrate or transferrin, which firmly binds aluminum at sites unoccupied by iron, and it appears to be responsible for the accumulation of the ion both in cells and tissues [4-7].

From the medical standpoint, aluminum and its salts are used in antiacids, antidiarrheals and protective dermatological pastes, as well as in cosmetics and deodorants. High doses of aluminum may originate different pathologies: aluminum intoxication with severe osteomalacia [8] and extensive aluminum deposition at the junction, between calcified and not-calcified bone, has been reported in several young children, who were receiving large dosages of aluminum hydroxide for the management of hyperphosphatemia associated with azotemia [9]. Aluminum salts may induce phosphorus depletion, which however is generally not severe, even though a prolonged administration of large doses may bring about hypophosphatemia, especially in patients with inadequate dietary intake of phosphorus; in these patients, hypercalciuria results as a secondary effect to bone resorption and increased intestinal absorption of calcium [10]. Intracranial administration of aluminum salts to cats, rabbits and dogs induces intraneuronal accumulations of neurofibrillary consisting of $10 \mathrm{~nm}$ intermediate filaments $[11,12]$ in perikarya and proximal axons which closely resemble neuronal alterations at the early stages of amyotrophic lateral sclerosis (ALS) and share some features with the neuronal lesions in the ALS-Parkinson dementia complex of Guam $[8,13,14]$. Aluminum at non-toxic doses is thought to be also involved in different diseases: in fact, excessive dietary aluminum has been proposed to be a factor contributing to several neurological disorders in humans. In particular, aluminum accumulation in the central nervous system (CNS) may be at the origin of dialysis dementia, which sometimes occurs in chronic renal failure patients receiving long-term aluminum antiacid therapy for hyperphosphatemia [15]. Recent epidemiological evidence associates increased bioavailability of aluminum with the incidence of Alzheimer's disease (AD) [1,5], and in this respect several 'in vitro' studies have demonstrated the neurotoxicity of the metal [7]. As a matter of fact, increased levels of aluminum have been observed in neuritic deposits, the plaques and the neurofibrillary tangles of patients affected by AD [16] and ALS [17].

However, despite the 'in vivo' and 'in vitro' evidence, no initial event(s) for the involvement of aluminum in AD or ALS has been established. Therefore, some researcher has questioned the importance of the role of aluminum in the early pathogenesis of these neurological disorders. Other investigations suggested that aluminum ions could be a factor for biological toxicity contributing to the aetiology of a variety of neurological and skeletal disorders, even though the molecular mechanisms underlying these processes are still unclear.
Recently, it has been reported that exposition to aluminum (also reported as environmental neurotoxin) can induce important alterations in signal transduction pathway [18] and that this effect may be associated either with the interaction with specific membrane lipids or with phosfatidylinositol-4,5-biphosphate, an element of the phosphoinositide signalling system even in the presence of high concentrations of $\mathrm{Ca}^{2+}$ [19]. The data reported indicate that the aluminum toxicity is achieved through a complex mechanism involving the interaction with specific metal binding sites on the enzyme molecules but also with specific membrane lipids (see above) [20]. The debated role of aluminum in the genesis of different pathologies in man, has seen involved also a possible influence of the ion on a correct efficiency of the proteolytic systems (i.e. protease and antiprotease activities) which has been, often, indicated as a possible pathogenic factor for many diseases. In fact, an increase of the cerebral proteases level (in particular the chymotrypsin- and trypsin-like serine proteases) apparently involved in the accumulation of $\beta$-amyloid peptides and tau proteins in AD has been reported [21]. Similarly, increased levels of calpain which appears involved in cytoskeletal pathologies and neurofilament accumulation associated with $\mathrm{AD}$ and aged brains has been observed $[21,22]$. In addition, imbalance in the dynamic equilibrium between proteases and their specific inhibitors has been invocated for the genesis of several pathologies included malignancies and neurological disorders and the modulative effect exerted by metallic ions on proteolytic systems has been recently emphasized. It has been reported that several mono- and divalent cations, particularly sodium and calcium, bind proteolytic enzymes, inducing conformational changes which affect the catalytic activity of proteinases and/or the recognition process by their protein inhibitors [2325]. On the other hand it has been shown that several peptides, upon addition of $\mathrm{Al}^{3+}$, undergo a conformational transition from $\alpha$-helix (or random coil) to $\beta$ pleated sheet before that their precipitation occurs following a molecular mechanisms which has been invocated also for the formation of senile plaques 'in vivo'. In addition, conformational studies on aluminum-trypsin complexes show that the ion influence the secondary structure of the enzyme causing a decrease in the $\alpha$-helix and an increase in the percentage of $\beta$-sheet and random coil conformations [26-28], before causing its precipitation [29-31].

These data support the idea that the functional behaviour of proteolytic enzymes could be modulated by environmental factors opening an additional field of investigation on this class of enzymes which are involved in a wide range of physiological processes and that are retained responsible for the generation of several pathological disorders in man. This paper concerns with the effects of aluminum (essentially $\mathrm{Al}^{3+}$ ) on 
different proteases (mainly $\alpha$-chymotrypsin and calpains), on their activity and/or specificity against natural substrates or inhibitors, indicating the role of this ion on the structure-function relationships of proteolytic enzymes.

\section{Effects of aluminum on the biological activity of serine proteinases}

Serine proteases are a wide group of endopeptidases, which are characterized by the presence of a serine residue at the active site. They are, certainly, the best characterized family of proteases and include the digestive enzymes trypsin, chymotrypsin, elastase and kallikrein. The effect of aluminum on the activity of serine proteases has been studied for several enzymes (see below) with particular emphasis to $\alpha$-chymotrypsin. This one, is a serine endopeptidase, which is mainly secreted from pancreas and represents the second major proteinase component of the pancreatic juice. It is produced as inactive form ( $\alpha$-chymotrypsinogen) by the acinar cells of pancreas and then carried as inactive enzyme by the pancreatic juice into the duodenum where it is activated by trypsin. Three amino-acid residues, His 57, Asp 102 and Ser 195, form the catalytic triad, as in all other serine proteases, and they are essential for peptide bond cleavage. The catalytic triad is located at the entrance of a substrate-binding pocket and a conservative structural arrangement is stabilized by hydrogen bonds.

Aluminum strongly influences the binding properties of $\alpha$-chymotrypsin, affecting both the substrate and the inhibitor(s) recognition process. The interaction between the ion and the enzyme has been explored either through fluorimetric measurements or studying the effect of the aluminum on the hydrolytic activity of the enzyme towards the synthetic substrate $N$ - $\alpha$-benzoyl-L-tyrosine ethyl ester (BTEE). The experimental data clearly demonstrate that the modulative effect is due to a simultaneous occupancy of the substrate and metalbinding sites and that the substrate binding brings about a modification of the ionic environment of the enzyme molecule, favouring aluminum binding. In particular, the aluminum does not affect the catalytic rate constant $k_{\text {cat }}$ (or the apparent value of $V_{\max }$ ), but it acts as an enzyme activator favouring the binding of the substrate to the catalytic site (i.e. decreasing $K_{\mathrm{M}}$ ) [32].

Furthermore, aluminum binding to $\alpha$-chymotrypsin induces a threefold decrease of the affinity of the enzyme for the macromolecular protein inhibitor bovine pancreatic trypsin inhibitor (BPTI) [32]. In addition, the negative heterotropic effect exerted by aluminum on the BPTI interaction with $\alpha$-chymotrypsin suggests that metal binding brings about a conformational change of the overall enzyme molecule, facilitating the interac- tion at the substrate primary specificity site, where the synthetic substrate binds, but decreasing the overall free energy of the secondary specificity site where only BPTI interacts. Altogether, the different effect of aluminum on the binding of synthetic substrates (e.g. BTEE) and macromolecular inhibitors (e.g. BPTI) to $\alpha$-chymotrypsin suggests the occurrence of an aluminum-linked conformational change in the enzyme molecule, which brings about a marked structural change at the primary and secondary recognition sites for substrates and inhibitors. In addition, the ion-linked effect appears to be dependent on the $\mathrm{pH}$ in a complex fashion, which suggests that aluminum binding is controlled by the protonation of at least two classes of residues on the enzyme molecule [32]. Thus, it appears that the modulative effect exerted by the metal on chymotrypsin-like proteolytic activities is mediated by a concerted series of events, which involves the metal and the substrate (as well as the inhibitor) binding and protonation phenomena occurring on the enzyme molecule.

An overall activating effect, determined by aluminum, was recently observed also on the proteolytic processes mediated by oxidized forms of $\alpha$-chymotrypsin [33]. Exposure of the native enzyme to hydrogen peroxide leads to the formation of mono- and di-methionine sulfoxide $\alpha$-chymotrypsin, which reasonably mimics for aged forms of the proteolytic enzyme. Oxidation of methionine residues apparently does not produce significative effects on the enzyme structure as well as on the functional properties. Binding of aluminum to both the mono- and dioxidized forms of the enzyme results in a significative activation (see Fig. 1B), which is very similar to that observed with the native enzyme (see Fig. $1 \mathrm{~A}$ and [32]). The activating effect on the proteolytic processes mediated by oxidated forms of $\alpha$-chymotrypsin is further strengthened by a protection, of the modified enzyme, from a rapid inactivation by macromolecular inhibitors, with particular reference to BPTI (Fig. 2B), suggesting that the role of aluminum could be very important in cellular conditions favouring protein oxidation such as degenerative diseases and aging. The protective effect exerted by the metal from the inhibitory action of protein inhibitors was observed also for the system $\alpha$-chymotrypsin/ $\alpha_{1}$-antichymotrypsin [34].

Aluminum appears to affect also the activity of trypsin as well as of other serine proteases such as Cathepsin G and porcine pancreas elastase (Lupidi et al., unpublished) even though the data reported appear ambiguous, at least for trypsin. Zatta et al. [35] reported the inhibitory effect exerted by $\mathrm{Al}^{3+}$-complexes on trypsin (and chymotrypsin). These results were accounted for a direct interaction of $\mathrm{Al}^{3+}$ with the carboxyl group of Asp 102, which forms the enzyme catalytic triad together with His 57 and Ser 195 (see above). By contrast Clauberg and Yoshi [34] reported an 

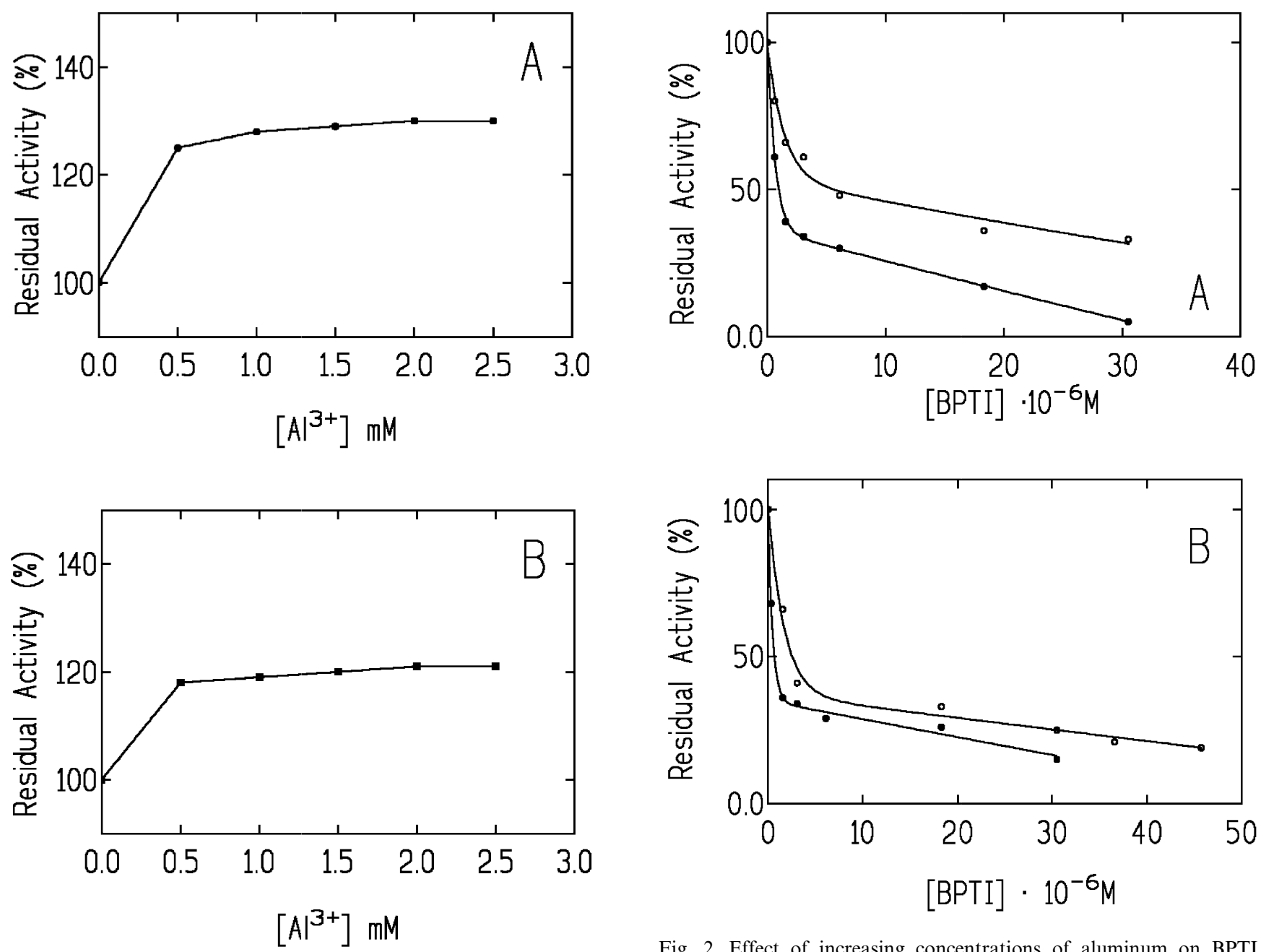

Fig. 1. Effect of increasing concentrations of aluminum on the activity of native (A) and mono-methionine sulfoxide (B) $\alpha$-chymotrypsin. The enzyme activity was determined following at $256 \mathrm{~nm}$ and $25{ }^{\circ} \mathrm{C}$, the hydrolysis of the synthetic substrate BTEE, in $50 \mathrm{mM}$ MOPS $-\mathrm{NaOH}$ at $\mathrm{pH}$ 6.5. When aluminum was added, as $\mathrm{AlCl}_{3}$, to the assay, 5-min pre-incubation of the enzyme with the metal was performed. The oxidized form of $\alpha$-chymotrypsin was obtained treating the native enzyme with $\mathrm{H}_{2} \mathrm{O}_{2}$ in $1 \mathrm{mM} \mathrm{HCl}$ [33].

activating effect exerted by aluminum on the same enzyme.

The 'in vivo' significance of this finding can be accounted for by the potential role ascribed to chymotrypsin-like proteases in brain metabolism, with particular reference to the aetiology of $\mathrm{AD}$ and to the debated role of aluminum in the deregulation of the brain proteolytic processes, leading to the generation of senile plaques, which are the major hallmark of this neurological disorder [36,37]. In this context the aluminum role could be envisaged at different levels. First, the metal could affect the formation of amyloid peptides through a positive modulation of the proteolytic processes involved in the $\beta$-amyloid precursor processing; then it could bind to the so-formed peptides, promoting

Fig. 2. Effect of increasing concentrations of aluminum on BPTI binding to the native (A) and mono-methionine sulfoxide (B) $\alpha$ chymotrypsin. When the effect of aluminum was studied, a solution of the enzyme (native or oxidized) in $50 \mathrm{mM}$ MOPS- $\mathrm{NaOH}$ at $\mathrm{pH} 6.5$ was incubated for $5 \mathrm{~min}$ with increasing amounts of the ion, added as $\mathrm{AlCl}_{3}$. A solution of BPTI was then added and, after an additional 5 min of incubation, the residual activity of $\alpha$-chymotrypsin was measured as reported in Fig. 1. The oxidized form of $\alpha$-chymotrypsin was obtained as indicated in Fig. 1.

their aggregation and precipitation and accelerating the formation of the neuritic plaque [38]. On the whole, aluminum produces an overall activating effect on $\alpha$ chymotrypsin, which can be observed at physiological concentration of the metal (estimated to be about 10 $\mu \mathrm{M}$ ), thus reinforcing the idea of a key role of this ion in different pathological processes, such as the formation of amyloid plaques. It must be underlined the role of $\mathrm{pH}$ in the activity of the aluminum, since it is well known that the bioavailability of aluminum is strongly influenced by $\mathrm{pH}$ and that some degenerative phenomena occurring, for example, at cerebral level (with particular reference to $\mathrm{AD}$ ) are accompanied by $\mathrm{pH}$ changes in some brain regions [39]. 


\section{Effect of aluminum on the binding properties of calpains}

Calpain is an intracellular, $\mathrm{Ca}^{2+}$-dependent cysteine proteinase that is widely distributed and it shows a regulated activity at neutral $\mathrm{pH}$. In mammals, two major ubiquitous forms of calpain have been characterized, namely $\mu$ - (calpain I) and m-calpain (calpain II). As suggested by the name $\mu$ - and m-calpains are activated 'in vitro' by micro- and millimolar concentrations of $\mathrm{Ca}^{2+}$, respectively. Each of these calpains consists of two subunits, a large one (ca. $80 \mathrm{kDa}$ ), which differs between the two calpains, and a small one (ca. $30 \mathrm{kDa}$ ), which is common to all calpains. The large subunit can be divided into four domains: the second and the fourth one correspond to the cysteine protease activity and to the $\mathrm{Ca}^{2+}$ binding domain, respectively, whereas the function of the first and of the third domain is presently unclear. The small subunit is composed of two domains, the N-terminal Gly-clustering, hydrophobic domain, and a $\mathrm{C}$-terminal $\mathrm{Ca}^{2+}$ binding region similar to that of the large subunit. The role of the small subunit is to regulate the activity of calpain. The two subunits are associated through the $\mathrm{Ca}^{2+}$ binding domains in the absence of $\mathrm{Ca}^{2+}$. Although there is still uncertainty about the role of calpains, it has been suggested that these enzymes may be involved in relatively specific cellular processes, such as regulation of cytoskeleton and myofibril assembly. Various pathological states, likely involving calpains, have been reported, including cataract formation, $\mathrm{AD}$, ischemia, inflammation and muscular dystrophy.

Several lines of evidence indicate a direct effect of aluminum salts on calpain. Aluminum inhibits calpainmediated proteolysis when azocasein, a substrate not appreciably altered by exposure to the ion, was used. Exposure of calpain alone to aluminum salts irreversibly reduced its activity, and the degree of inhibition increased with the time of exposure to the metal [11]. Since aluminum has a high affinity for calcium-binding proteins and enzymes [40], this inhibitory effect may be partially related to multiple calcium binding domains on the two calpains subunits [41]. Calpain-mediated proteolysis of human neurofilament subunits (NF-H, NF$\mathrm{M}$ and NF-L), tubulin and glial fibrillary acidic protein (GFAP) was inhibited by $\mathrm{AlCl}_{3}\left(\mathrm{IC}_{50}=200 \mu \mathrm{M}\right)$ and by aluminum lactate $\left(\mathrm{IC}_{50}=400 \mu \mathrm{M}\right)$. In these cases it should be underlined that aluminum salts might act by directly affecting the substrate. In any case, aluminum salt irreversibly inactivated calpain but only at high aluminum concentrations $\left(\mathrm{IC}_{50}=1.2\right.$ and $2.1 \mathrm{mM}$ for aluminum lactate and $\mathrm{AlCl}_{3}$, respectively), although a longer exposure to the ion reduced by twofolds the level required for protease inhibition. The interaction of aluminum with neurofilament proteins and the effects on their proteolysis [12], suggest possible mechanisms for the impaired axoplasmic transport of neurofilaments and their accumulation in neuronal perikarya after aluminum absorption 'in vivo'. Other studies confirmed these results: in the presence of millimolar concentrations of $\mathrm{Ca}^{2+}$ calpain I was inhibited by submillimolar concentrations of aluminum ion $\left(\mathrm{IC}_{50}=100 \mu \mathrm{M}\right)$; but in the millimolar range aluminum ion was a less effective inhibitor and the activity of the enzyme reached about $65 \%$ (at a concentration of aluminum of $10 \mathrm{mM}$ ) of the control activity assayed without aluminum.

By contrast, calpain II displays a progressive inhibition by aluminum ion with an $\mathrm{IC}_{50}$ value of $2.4 \mathrm{mM}$ [42]. These results suggest that in the case of calpain I there might be different binding sites for aluminum ion on the enzyme, with occupancy of site(s) in the micromolar range inducing inhibition while occupancy of other sites in the low millimolar range having then an activator effect on the enzyme. Another difference between the effects of aluminum ion on calpains I an II was that in the absence of $\mathrm{Ca}^{2+}$, calpain II could be activated to ca. $60 \%$ of the $\mathrm{Ca}^{2+}$ activated activity level at low millimolar aluminum ion concentrations [43]. As this activating effect is abolished at higher aluminum ion concentrations, the existence of multiple binding sites for aluminum ion on calpain II also seems plausible. When calpain II is activated in presence or absence of aluminum ion the proteolytic specificity results almost identical. It should be underlined that the $\mathrm{Ca}^{2+}$ activated calpain undergoes a rapid autolysis, whereas the enzyme activated in the presence of aluminum ions did not autolyse to any detectable extent, retaining its proteolytic activity over a long period of time. This difference suggests that different conformational changes may occur to the enzyme as a result of the binding of the two different metal ions. On the whole, the modulation of the activity of calpain by aluminum ions might involve complex mechanisms or different pathways that reduce the overall activity of these enzymes crucial for the cytoskeleton and cellular behaviour.

\section{Effect of aluminum on the proteolytic enzymes of cerebral cortex}

Aluminum also affects the biological activity of several cytoplasmic and lysosomial proteases [44] localized in human cerebral cortex. A systematic study performed with a series of potentially neurotoxic metals [45] shows that aluminum at $5 \mathrm{mM}$ concentration, inhibits the activity of several cytoplasmic peptidases (alanyl-, arginyl-, leucyl-, dipeptidyl- tripeptidyl- and proline-amino peptidase) with the exception of pyroglutamyl aminopeptidase which results are activated. Similarly, the ion at $5 \mathrm{mM}$ concentration, inhibits several lysosomial proteinases (Cathepsin B, L and $\mathrm{H}$ 
and dipeptidyl aminopeptidase I and II) while it enhances the activity of Cathepsin D. In the same study the inhibitory effect of aluminum on Calpains I and II partially purified from the same source (see also above) was also shown. These effects were linked to the ability of aluminum to alterate the protein turnover in the brain and envisaged as potential pathogenic agents for neurological disorders with particular reference to the AD. However, it must be outlined that the concentration of the metal affecting the proteolytic activities appears larger than the mean value of the metal found in normal or pathological tissues of the CNS as whole. This does not allow to exclude 'a priori' that aluminum levels could increase in particular regions of the tissue owing to specific metal compartmentalization or deposition processes in particular associated to pre- or pathological conditions.

\section{Concluding remarks}

The data discussed here clearly indicate that aluminum ions affect the biological activity of proteolytic activities either through a direct effect on the enzyme molecule or through a modification of the regulatory mechanisms acting on them. From a molecular point of view the effect appears to be the consequence of molecular rearrangements occurring on the enzyme molecule upon metal binding. The physiopathological significance of this effect could be questionable since it appears only an early event affecting the intracellular protein turnover; however, since an enhanced degradative metabolism of proteins appears to involve also the physiological aluminum carriers (i.e. transferrin or albumin) it could be envisaged as the trigger of a series of catastrophic events for the cellular life (including deposition of aluminum, enhancement of free radicals production etc.). The data reported indicate also a certain ambiguosity which can be accounted for by both the complex chemistry of aluminum (with particular reference to the 'in vivo' speciation of aluminum, which remains an open question) and the experimental approach utilized since some results were obtained on purified systems some others on crude extracts of tissue or organs; some of them refer to a quantitative analysis of the phenomena observed, others refer only to qualitative effects. In any case, all the experimental evidence lead to the idea that aluminum is a toxic metal for biological systems. This etiopathogenic role could be further stressed in neuronal disorders with a higher emphasis for the AD where an imbalance of proteolytic as well as antiproteolytic systems appears a crucial event both for the formation of neuritic plaques and neurofibrillary tangles which are the major hallmarks of the disease. Furthermore, neurologic disorders are often accompanied by increased rates of lipid peroxidation in the brain and aluminum appears able to enhance the intracellular oxidative phenomena [46] through an enhancement of the iron catalyzed, oxygen-derived free radical production [47] which in turn can affect both proteins oxidation (and then their turnover) or membrane lipid peroxidation [48] (and then membrane integrity and efficiency). Similar conclusions can be achieved from 'in vivo' experiments showing that increased levels of lipid peroxidation in animals treated with aluminum are paralleled by a decrease of the activity of antioxidant enzymes such as superoxide dismutase, catalase and glutathione peroxidase [49]. Moreover, it has been reported that several proteases are induced by the presence of oxidatively damaged proteins $[50,51]$ and that aluminum promotes prooxidant events in the brain [52]. A particular importance in this scenario must be attributed to the intracellular $\mathrm{pH}$ value which appears slightly acidic in the case of the $\mathrm{AD}$ brains. In this condition, the formation of the oxygen free radicals as well as the activating effect exerted by aluminum on the proteolytic activities (at least for serine proteases) appear enhanced leading to an overall increase of the protein degradation processes and then to an increased production of neurotoxic peptides, $\beta$ amyloid included.

\section{Acknowledgements}

The financial support from Fondazione Cassa di Risparmio di Fabriano e Cupramontana to this work is gratefully acknowledged.

\section{References}

[1] R.B. Martin, Clin. Chem. 32/10 (1986) 1797.

[2] T. Macdonald, R.B. Martin, Trends Biochem. Sci. 13 (1988) 15.

[3] S.A. Fatemi Jemil, D.J. Williamson, G.R. Moore, J. Inorg. Biochem. 46 (1992) 35.

[4] B.R. Martin, J. Inorg. Biochem. 28 (1986) 181.

[5] R.B. Martin, J. Savory, S. Brown, R.L. Bertolf, M.R. Wills, Clin. Chem. 33 (1987) 405.

[6] C.N. Martyn, D.J.P. Barker, C. Osmond, E.C. Harris, J.A. Edwardson, R.F. Lacey, Lancet (1989) 59.

[7] J.G. Joshi, Bio Factors 2 (1990) 163.

[8] A.C. Alfrey, in: M. Niccolini, P.F. Zatta, B. Corain (Eds.), Aluminum in Chemistry Biology and Medicine, Raven, New York, 1991, pp. 73-84.

[9] S.P. Andreoli, D. Dunn, W. DeMyer, D.J. Sherrard, J.M. Bergstein, J. Pediatr. 107 (1985) 760.

[10] M. Davies, M.R. Clements, E.B. Mawer, A.J. Freemont, J. Med. 63 (1987) 493.

[11] R.A. Nixon, J.F. Clarke, K.B. Logvinenko, M.K.H. Tan, M. Hoult, F.J. Grynspan, Neurochemistry 55 (1990) 1950.

[12] T.B. Shea, P. Balikian, M.L. Beermann, FEBS Lett. 307 (1992) 195.

[13] H. Wisniewski, R.D. Terry, A. Hirano, J. Neuropathol. Exp. Neurol. 29 (1970) 163.

[14] P.F. Zatta, Trace Elem. Med. 10 (1993) 120. 
[15] M.R. Wills, J. Savory, Crit. Rev. Clin. Lab. Sci. 27 (1989) 59.

[16] D.R. Crapper-McLachlan, Neurobiol. Aging 7 (1986) 525.

[17] D.P. Perl, D.C. Gajdusek, R.M. Garruto, R.T. Yanigihara, C.J. Ggibbs Jr., Science (1982) 1053.

[18] M. Llansola, M.D. Minana, C. Montoliu, R. Saez, R. Corbalan, L. Manzo, V. Felipo, J. Neurochem. 73 (1999) 712.

[19] L.J. McDonald, M.D. Mamrack, J. Lipids Mediators Cell Signal. 11 (1995) 81.

[20] D.L. Jones, L.V. Kochian, FEBS Lett. 400 (1997) 51.

[21] S. Guo-Ross, E. Yang, S.C. Bondy, Int. Neurochem. 33 (1998) 277.

[22] M. Banay-Schwartz, T. DeGuzman, M. Palkovits, A. Lajtha, Neurochem. Res. 19 (1994) 563.

[23] C.M. Wells, E. Di Cera, Biochemistry 31 (1992) 11721.

[24] M. Nayal, E. Di Cera, Proc. Natl. Acad. Sci. USA 91 (1994) 817.

[25] E. Fioretti, M. Angeletti, G. Lupidi, M. Coletta, Eur. J. Biochem. 225 (1994) 459.

[26] V. Di Noto, L. Dalla Via, P. Zatta, J. Raman Spectrosc. 30 (1999) 209.

[27] Z.M. Shen, A. Perczel, M. Hollosi, I. Nagypal, G.D. Fasman, Biochemistry 33 (1994) 9627.

[28] I. Laczko, E. Vass, K. Soos, J.L. Varga, S. Szaraz, M. Hollosi, B. Penke, Arch. Biochem. Biophys. 335 (1996) 381.

[29] M. Hollosi, L. Urge, A. Perczel, J. Kajtar, I. Teplan, L. Otvos, Jr., G.D. Fasman, J. Mol. Biol. 223 (1992) 673.

[30] M. Hollosi, L. Otvos, Jr., L. Urge, J. Kajtar, A. Perczel, I. Laczko, Z.S. Vadasz, G.D. Fasman, Biopolymers 33 (1993) 497.

[31] H. Inouye, P.E. Fraser, D.A. Kirchner, Biophys. J. 64 (1993) 502.
[32] M. Angeletti, G. Lupidi, A.M. Eleuteri, R. Tacconi, E. Fioretti, M. Coletta, J. Biol. Inorg. Chem. 2 (1997) 320.

[33] G. Lupidi, M. Angeletti, A.M. Eleuteri, E. Fioretti, M. Coletta, (2002) submitted.

[34] M. Clauberg, J.G. Yoshi, Proc. Natl. Acad. Sci. USA 90 (1993) 1009.

[35] P. Zatta, C. Bordin, M. Favarato, Arch. Biochem. Biophys. 303 (1993) 407

[36] R.B. Nelson, R. Siman, J. Biol. Chem. 265 (1990) 3836.

[37] B.L. Razzaboni, G. Papastoitsis, E. Koo, C.R. Abraham, Brain Res. 589 (1992) 207.

[38] S.B. Vyas, L.K. Duffy, J. Protein Chem. 14 (1995) 633.

[39] C.M. Yates, J. Butterworth, M.C. Tennant, A. Gordon, J. Neurochem. 55 (1990) 1624.

[40] E. Habermann, G. Richardt, Trends Pharmacol. Sci. 7 (1986) 298.

[41] K. Aaoki, S. Imajoh, S. Ohno, Y. Emori, M. Koike, G. Kosaki, K. Suzuki, FEBS Lett. 205 (1990) 313.

[42] H. Zhang, P. Johnson, Int. J. Biochem. 24 (1992) 1773.

[43] D.R. Crapper, S.S. Krishnan, Brain 99 (1976) 67.

[44] C.B. Dobson, J. Graham, R. Itzaki, Exp. Neurol. 153 (1998) 342.

[45] G. Falkous, B.J. Harris, D. Mantle, Clin. Chim. Acta 238 (1995) 125.

[46] A. Campbell, S.C. Bondy, Cell. Mol. Biol. 46 (2000) 721.

[47] S.C. Bondy, S. Kirstein, Mol. Chem. Neuropathol. 27 (1996) 185.

[48] T. Ohyashiki, S. Suzuki, E. Satoh, Y.A. Uemori, Biochim. Biophys. Acta 1389 (1998) 141

[49] D. Julka, K.D. Gill, Res. Exp. Med. 196 (1996) 187.

[50] R.T. Dean, FEBS Lett. 220 (1987) 278.

[51] K.J.A. Davies, Biochem. Soc. Trans. 21 (1993) 346

[52] S.C. Bondy, S.F. Ali, S. Guo-Ros, Neurochem. Int. 33 (1998) 51. 